\title{
The impact of laser shock waves on anodic oxide - compound semiconductor interface
}

\author{
V.S. Yakovyna ${ }^{1}$, N.N. Berchenko' ${ }^{1}$, Yu.N. Nikiforov ${ }^{2}$ \\ 1 - Lviv Polytechnic National University, 12 Bandera St., 79013 Lviv, Ukraine \\ phone +38 (0322) 398-627, fax +38 (0322) 744-300, email yakovyna@polynet.lviv.ua \\ 2 - Ternopil State Technical University, 56 Russka St., 46001 Ternopil, Ukraine
}

\begin{abstract}
The present work shows that the induced positive charge value at the anodic oxide $-\mathrm{HgCdTe}$ (AO-MCT) interface reaches its constant value $(6.0 \pm 1.0) \cdot 10^{11} \mathrm{~cm}^{-2}$ under laser shock wave (LSW) treatment. We demonstrate that this treatment decreases electron concentration in a converted $n$-type layer by one order of magnitude in annealed AO-MCT based structures, and the annealing time needed to have this layer disappeared decreases as well. The model of LSW impact on the AO-MCT interface is analysed.
\end{abstract}

Keywords: shock wave, $\mathrm{HgCdTe}$, anodic oxide, interface.

Paper received 05.10.01; revised manuscript received 28.11.01; accepted for publication 12.12.01.

\section{Introduction}

In contrast to silicon, intrinsic oxides have not been widely used to protect compound semiconductors-based devices yet. An anodic oxide (AO) seems an exclusive example, and it is used to passivate $\mathrm{HgCdTe}$ (MCT) based photoconductive detectors $[1,2]$. However, AO-based structures have poor thermal stability. At temperatures above $100^{\circ} \mathrm{C}$ a $n$ type layer is formed at a semiconductor interface, with thickness and carrier concentration increasing up to a certain value as temperature and annealing duration increasing [3, 4]. HgZnTe-based AO structures [5, 6] demonstrate better thermal stability, but current state of the art does not allow to fabricate high-quality material for wide application. That is why techniques to control interface characteristics and AO-MCT structures thermal stability should be developed. The laser shock wave (LSW) treatment could be an adequate approach.

Recently we have studied the LSW impact on bulk MCT single crystals both $n$ - and $p$-type [7]. The results we obtained show that LSW-induced gettering of native point defects on different sinks is a major contributor that is in a good agreement with [8]. Since properties of crystals with macroinhomogeneties were the most changed, we can expect parameters of the AO-MCT interface with its well known structure $[3,9]$ to be changed considerably as well.

Thus, our study focuses of the LSW impact on the AOMCT interface properties and thermal stability.

\section{Experiment}

Neodymium-glass laser pulses with $1.06 \mathrm{~mm}$ wavelength, $30 \mathrm{~ns}$ pulse width, were used to induce a shock wave in the samples at room temperature. A $100 \mathrm{~mm}$ copper foil was used as an opaque overlayer to protect samples from the thermal influence of the laser beam. Dimensions of samples to be treated were chosen to assure the shock compression to be uniform and uniaxial (typical dimension is $5 \times 6 \times 1 \mathrm{~mm}^{3}$ ).

The samples were anodised in $0.1 \mathrm{M} \mathrm{KOH}, 90 \%$ ethylene glycol $10 \%$ deionised water electrolyte under $0.3 \mathrm{~mA} \cdot \mathrm{cm}^{-2}$ controlled current conditions, and the anodization terminated after the voltage drop had reached $15 \mathrm{~V}$. This voltage drop value corresponds to the oxide thickness of $800 \AA$.

Each and every sample was cut out of the same $p$-type MCT wafer with $x \approx 0.20-0.22$ and the initial carrier concen- 


\section{V.S. Yakovyna et al.: The impact of laser shock waves on anodic oxide...}

tration of (6-8) $10^{15} \mathrm{~cm}^{-3}$. The Hall measurements were carried out in variable magnetic fields up to $1.5 \mathrm{~T}$ at $77 \mathrm{~K}$ to determine carrier parameters using the Petritz model. Low $p$-type samples were chosen because it is easy to fix even a little contribution of converted layer electrons to $R_{H}(B)$ dependencies in such a material.

Three groups of samples were studied. The processing sequence used in experiments with the group 1 samples included anodization and annealing in ambient air at $150{ }^{\circ} \mathrm{C}$ immediately after anodization without LSW treatment. In contrast, the group 2 samples were annealed under the same conditions after anodization and LSW treatment of the back (not oxidised) sample surface. And finally the group 3 samples were treated in the following way - anodization, LSW treatment (LSW enters the anodized surface) and annealing as it is described above.

The annealing period varied from one to nine hours. After annealing the anodic oxides were removed by etching in a weak aqueous solution of hydrofluoric acid.

\section{Results and discussion}

First of all the influence of anodization on electrical properties of initial samples was considered. It was established that the anodization results in Hall coefficient sign conversion in weak fields. This fact is concerned with the fixed positive charge formation. From the analysis of $R_{H}(B)$ one can determine the carrier parameters in $n$-type layer induced near the interface. The typical values of areal electron density are $5.0 \cdot 10^{11} \div 1.1 \cdot 10^{12} \mathrm{~cm}^{-2}$ and their mobility is within $18000-22000 \mathrm{~cm}^{2} /(\mathrm{V} \cdot \mathrm{s})$ that is in a good agreement with data obtained before $[1,2,10,11]$. It was also determined that anodization of $p$-type samples followed by removing
AO does not affect the Hall coefficient field dependence. Thus, without subsequent annealing anodization creates only fixed charge at interface and there is no evidence of the converted $n$-type layer in the semiconductor volume. This proves the fact that thermal energy at room temperature is insufficient to cause mercury diffusion from the source at the interface.

Since in present investigation AO-based structures should be annealed at rather high temperature the influence of such thermal treatment on carrier parameters of $p$ type bulk MCT have been studied. It was ascertained that the hole density in initial samples without AO slightly changes from $6 \cdot 10^{15}$ to $8 \cdot 10^{15} \mathrm{~cm}^{-3}$ after the 9 hours annealing at $150{ }^{\circ} \mathrm{C}$. Such changes of hole concentration don't affect the electron density determined from the $R_{H}(B)$ dependencies analysis. Thus, since samples used in current investigation were passivated with $\mathrm{AO}$ which prevent mercury outdiffusion, the influence of annealing on hole density will be significantly less.

It have been determined that the LSW treatment and subsequent oxide removing without any heat treatment does not affect the Hall coefficient field dependencies. So the LSW passing through the interface changes only positive fixed charge and does not create any $n$-type layer in the MCT volume.

Therefore let us discuss the LSW impact on the interface and fixed charge at it.

The areal electron density and their mobility for the group 3 samples are listed in Table 1 for every processing step.

As it is well known and described above the anodization create positive fixed charge at the interface. This charge causes thin induced $n$-type layer to be formed in the semi-

Table 1. Electron parameters taken at $77 \mathrm{~K}$ after every processing step

\begin{tabular}{lcc}
\hline \hline Step & $N, \mathrm{~cm}^{-2}$ & $\mu_{n}, \mathrm{~cm}^{2} /(\mathrm{V} \cdot \mathrm{s})$ \\
\hline Anodization & Sample $1-1$ & \\
\hline LSW treatment, $P_{\text {LSW }}=3 \times 0.785 \mathrm{GPa}$ & $1.07 \cdot 10^{12}$ & 18400 \\
\hline 2.25 hours annealing & $6.39 \cdot 10^{11}$ & 24600 \\
\hline AO removing & $6.12 \cdot 10^{11}$ & 58400 \\
\hline & $4.22 \cdot 10^{11}$ & 52800 \\
\hline Anodization & Sample $1-2$ & 20700 \\
\hline LSW treatment, $P_{\text {LSW }}=1.092 \mathrm{GPa} \times 0.783 \mathrm{GPa}$ & $4.99 \cdot 10^{11}$ & 22500 \\
\hline 1 hour annealing & $6.74 \cdot 10^{11}$ & 50700 \\
\hline AO removing & $5.58 \cdot 10^{11}$ & 93000 \\
\hline & $1.37 \cdot 10^{11}$ & 21800 \\
\hline Anodization & Sample $1-3$ & 20400 \\
\hline LSW treatment, $P_{\text {LSW }}=3 \times 0.656 \mathrm{GPa}$ & $6.59 \cdot 10^{11}$ & 51300 \\
\hline 4 hours annealing & $4.76 \cdot 10^{11}$ & 69600 \\
\hline AO removing & $4.61 \cdot 10^{11}$ & \\
\hline \hline
\end{tabular}




\section{V.S. Yakovyna et al.: The impact of laser shock waves on anodic oxide...}

conductor. The evidence of such a layer is well seen for each sample from the Table 1 after the first step. Note that the induced layer electrons correspond to "quasi-2D carriers" that can be easily seen from their low mobility. It should be mentioned also the sample-to-sample scatter of areal electron density which corresponds to fixed charge value diversity. This sample-to-sample diversity exists even though entire slice was subjected to anodization and then cut onto individual samples. Such a kind of scatter we ascribe to individual features of the samples such as surface and defect status.

As it is shown in Table 1 the value of areal electron density after the LSW treatment can both decrease (samples $1-1$ and $1-3$ ) and increase (sample $1-2$ ).

Such changes we first of all have attributed to LSW impact on positive fixed charge at the interface. We think after LSW treatment the charge reaches its constant value of $(6.0 \pm 1.0) \cdot 10^{11} \mathrm{~cm}^{-2}$ and this value depends on the each sample surface and defect status mentioned above. We state that obtained positive fixed charge value is an equilibrium one for each individual sample. Approaching to this interface equilibrium state can results in decreasing or increasing charge value, which in its turn results in corresponding changes of electron density. Apart as one can see from the Table 1 LSW treatment does not affect the electron mobility. This fact fairly agrees with the absence of any converted $n$-type layer in the samples after LSW treatment following by oxide removing without any heat treatment described above. That is a clear evidence of the electrons to be the same "quasi-2D carriers" of induced layer which is formed by $\mathrm{AO}$ itself without any other processing.

Now let us compare the behaviour of samples in all groups after annealing.

Carrier parameters for three groups of samples after final removing the oxide are listed in Table 2. The results for the group 1 samples qualitatively agree with those available in [4]. There are three regions distinct from each other, where:(1) $n$ grows rapidly at the start of annealing, (2) reaches its maximum value, and (3) decreases. P. Brogowski and J. Piotrowski [4] ascribed this decreasing to exhaustion of the mercury source after a prolonged annealing, resulting in mercury diffusion deeply into the bulk with reconversion of conductivity type. During annealing back side LSW treated samples we observed a slightly decrease of electron density, and its maximum was reached a little faster, after a shorter annealing (group 2 samples, Table 2). The same effects but much more significant we determined during annealing conventionally LSW treated samples of group 3. For example, at the minimum annealing time (1 hour) the conversion occurs with the electron density being less by one order of magnitude. This permits us to state that the conversion depth is also significantly less than that in the sample without LSW processing. While the annealing lasts up to 2.25 hours, the $n$ value continues growing in all groups of samples. After 4 hours annealing, however, the electron concentration decreases only in group 3 samples while in non-LSW-treated samples and in the back-side-LSW-treated one this happens much later. We ascribe this fact to the mercury source exhaustion reported in [4].

Large discrepancies of electron density in the samples of groups $2 \mathrm{i} 3$ we ascribe to some decay in LSW magnitude during its passing through the overall sample bulk in the case of the group 2 samples and except those with possible differences in LSW impact on the interface due to the pressure gradient being of the opposite sign.

Thus, within the AO-MCT interface model developed in $[3,11]$ the following factors are supposed to be responsible for overall changes in samples caused by LSW: an increased potential barrier that prevents the elemental mercury from interface-to-semiconductor moving; the elemental mercury removed from an interface into an oxide volume and replaced with HgTe-based complexes; the AO-MCT interface rebuilding, which decreases the free mercury concentration. Additionally, elemental mercury and tellurium may probably form $\mathrm{HgTe}$ complexes at the interface.

Table 2. Electron parameters at $77 \mathrm{~K}$ after annealing and subsequent oxide removing

\begin{tabular}{|c|c|c|c|c|}
\hline Sample & Annealing period, hours & LSW treatment & $n, \mathrm{~cm}^{-3}$ & $\mu_{n}, \mathrm{~cm}^{2} /(\mathrm{V} \cdot \mathrm{s})$ \\
\hline $1-1$ & 1 & - & $1.37 \cdot 10^{13}$ & 103000 \\
\hline $2-3$ & 1 & + & $1.21 \cdot 10^{13}$ & 111600 \\
\hline $3-2$ & 1 & + & $1.48 \cdot 10^{12}$ & 93000 \\
\hline$\overline{1-2}$ & 2.25 & - & $3.20 \cdot 10^{13}$ & 110700 \\
\hline $2-2$ & 2.25 & + & $1.96 \cdot 10^{13}$ & 120700 \\
\hline $3-1$ & 2.25 & + & $4.63 \cdot 10^{12}$ & 52800 \\
\hline $1-3$ & 4 & - & $4.39 \cdot 10^{13}$ & 106300 \\
\hline $2-4$ & 4 & + & $4.36 \cdot 10^{13}$ & 111600 \\
\hline $3-3$ & 4 & + & $2.18 \cdot 10^{12}$ & 69600 \\
\hline $1-4$ & 6.25 & - & $7.65 \cdot 10^{13}$ & 97900 \\
\hline $1-5$ & 9 & - & $6.94 \cdot 10^{13}$ & 110500 \\
\hline $2-1$ & 9 & + & $5.00 \cdot 10^{13}$ & 97700 \\
\hline
\end{tabular}


We believe that the most relevant mechanism of the AO-MCT interface-LSW interaction should involve the AO-MCT rebuilding which implies that:

- elemental mercury and related charge is pressed back from the $\mathrm{CdTeO}_{3}$ layer to the upper one;

- $\left(\mathrm{HgTe}+\mathrm{V}_{\mathrm{O}}\right)$-like complexes are formed under LSW impact;

- HgTe is accumulated at the interface.

Hence, newly formed complexes enlarge the induced charge. We suggest pressing back the mobile charge into the oxide bulk to be highly probable, and both the increase and decrease of the residual charge can happen depending on the removed - generated charge relation as it was found out with galvanomagnetic measurements. Secondly, such complexes prevents the elemental mercury from diffusing from the layer above. It is mainly due to moving the elemental mercury from the interface that the post-annealing electron concentration in LSW-treated samples is less than that in samples which were not exposed to LSW. There is a certain decrease of the fixed charge after annealing that may decrease the measured electron density. On our opinion, this can be explained by oxygen indiffusion resulting in filling oxygen vacancies in complexes responsible for the fixed charge. For annealing in the ambient air, Auger spectra indicate an increased oxygen content at the interface [9].

Besides, the mechanism we propose is well co-ordinated with the data on the LSW impact on Si-based structures with the $\mathrm{SiO}_{2}$ layer available at [12]. The results [12] indicate that LSW interacts with $\mathrm{SiO}_{2}$ and deforms $\mathrm{Si}-\mathrm{O}$ bonds, that promote the structure rebuilding of $\mathrm{SiO}_{2}$ surface layers, which, in turn, changes the structure of the dielectric throughout its volume and the structure of the $\mathrm{SiO}_{2}$ layer at the interface.

To confirm or discard this concept many advanced techniques should be used to study surface and elemental composition. As such techniques are not currently available, we are planning another research on the mechanism of the LSW-AO-MCT interface interaction.

\section{Conclusions}

1. The positive fixed charge at the AO - MCT interface is changed under LSW impact. This changes results in a certain equilibrium value of an fixed charge density, which varies within $(6.0 \pm 1.0) \cdot 10^{11} \mathrm{~cm}^{-2}$ depending upon a surface conditions and defect structure of a sample.
2. Electron density in a converted $n$-type layer formed in p-type MCT after annealing AO-based structures decreases by one order in magnitude in LSW-treated samples in comparison with that in samples that were not exposed to LSW.

3. The mechanism of the LSW and AO-MCT interface interaction is developed.

\section{References}

1. M.B. Reine, K.R. Maschhoff, S.P. Tobin, P.W. Norton, J.A. Mroczkowski and E.E. Krueger, The impact of characterization techniques on $\mathrm{HgCdTe}$ infrared detector technology // Semicond.Sci.Technol. 8, pp. $788-804$ (1993).

2. O.P. Agnihotri, C.A. Musca and L. Faraone, Current status and issues in the surface passivation technology of mercury cadmium telluride infrared detectors // Semicond.Sci.Technol. 13, pp. 839-845(1998).

3. C.M. Stahle, C.R. Helms, and A. Simmons, Thermal stability of HgCdTe anodic oxide // J.Vac.Sci.Technol. B5, pp. 1092-1100 (1987).

4. P. Brogowski and J. Piotrowski, The p-to-n conversion of $\mathrm{HgCdTe}, \mathrm{HgZnTe}$ and HgMnTe by anodic oxidation and subsequent heat treatment // Semicond.Sci.Technol. 5, pp. 530-532 (1990).

5. I. Esquivias, J. Baars, D. Brink and D. Eger, Electrical properties of the anodic oxide $-\mathrm{HgZnTe}$ interface // Semicond.Sci.Technol., 8, pp. S71-S74(1993).

6. K.H. Khelladi, D. Lemoine, S. Rolland, R. Granger and $\mathrm{R}$. Triboulet, Interface properties of passivated $\mathrm{HgZnTe} / /$ Semicond.Sci.Technol., 8, pp. 56-62 (1993).

7. N.N. Berchenko, V.S. Yakovyna, I.S. Virt, Yu.N. Nikiforov, On control the properties of bulk $\mathrm{Hg}_{1-\mathrm{X}} \mathrm{Cd}_{\mathrm{X}} \mathrm{Te}$ by laser induced shock waves // Proc. SPIE 4355, pp. 200-203 (2001).

8. V.A. Gnatyuk, A.I. Vlasenko, P.O. Mozol', and O.S. Gorodnychenko, Role of laser-induced stress and shock waves in modification of the photoconductivity of $\mathrm{Cd}_{\mathrm{x}} \mathrm{Hg}_{1-\mathrm{x}} \mathrm{Te}$ films // Semicond.Sci.Technol. 13, pp. 1298-1303 (1998).

9. C.M. Stahle, C.R. Helms, H.F. Shaake, R.L. Strong, A. Simmons, J.B. Pallix, C.H. Becker, Physical and chemical properties of the anodic oxide / HgCdTe interface // J.Vac.Sci. Technol. A 7(2), pp. 474-482 (1989).

10. Nemirovsky Y. and Bahir G., Passivation of mercury cadmium telluride surfaces // J.Vac.Sci.Technol. A 7(2), pp. 450-459 (1989).

11. R.B. Scholar, B.K. Janousek, R.L. Alt, R.C. Carscalen, M.J. Daughtery and A.A. Fote, Optical and field effect studies of the $\mathrm{Hg}_{0.7} \mathrm{Cd}_{0.3} \mathrm{Te}-$ anodic oxide interface // J.Vac. Sci. Technol. 21(1), pp. 164-167 (1982).

12. Y. Nikiforov, V. Yakovyna, N. Berchenko, Laser shock waves as a tool of changing strains in materials //Materials Science and Engineering $A$ 288(2), pp. 173-176 (2000). 